\title{
Sur trois Nématodes Trichostrongylides parasites de Tupaiidae
}

\author{
par M.-C. DURETTE-DESSET et A.-G. CHABAUD \\ Laboratoire de Zoologie (Vers) associé au C.N.R.S. \\ Muséum national d'Histoire naturelle, 43, rue Cuvier, F 75231 Paris Cedex 05
}

\section{Résumé.}

1) Description de Nycteridostrongylus (Petiellus) petersi n. sub. gen., n. sp., parasite de l'intestin et du poưmon de divers Tupaia à Borneo.

L'espèce type unique de Nycteridostrongylus, Baylis 1930, est parasite de Chiroptères en Australie et au Viet-Nam. Elle se différencie de celle du Tupaia par l'existence d'une dent œsophagienne dorsale et par un appareil de fixation cervical particulier.

2) Redescription d'Anoplostrongylus (Tupaiostrongylus) liei (Dunn, 1963) n. cb., parasite de divers Tupaia en Malaisie et à Bornéo. Les deux espèces connues jusqu'à présent dans le genre sont des parasites de Chiroptères sud-américains.

3) Redescription de Hepatojarakus malayae, Yeh, 1955, parasite de l'intestin de Tupaia tana (Tupaiidae) et d'Aeromys thomasi (Petauristinae) à Bornéo.

Il s'agit d'un Molineinae remarquable par la dilatation du corps et par le synlophe constitué de très nombreuses crénelures.

\section{Summary.}

On three Trichostrongylidae (Nematoda) parasite of Tupaiidae.

1) Description of Nycteridostrongylus (Petiellus) petersi n. sub. gen., n. sp., parasite of gut and lung of different Tupaia in Bornéo.

The single type species of Nycteridostrongylus Baylis, 1930, is parasite of Chiroptera in Australia and Viet-Nam. It differs from the species of Tupaia by the presence of a dorsal œsophagal tooth and by peculiar clasping apparatus in the cervical area.

2) Redescription of Anoplostrongylus (Tupaiostrongylus) liei (Dunn, 1963) n. cb., parasite of several Tupaia in Malaysia and Borneo. The two species known to date within the genus are parasite of South american Chiroptera. 
3) Redescription of Hepatojarakus malayae Yeh, 1955, parasite of the gut of Tupaia tana (Tupaiidae) and of Aeromys thomasi (Petauristinae) in Borneo.

This noticeable Molineinae stands out by the following characters: swelling of body, and numerous crenated cuticular ridges.

\section{DESCRIPTION DES ESPECES}

\section{Nycteridostrongylus (Petiellus) petersi n. sub. gen., n. sp.}

MATÉRIEL TYPE: 11 б, 23 \% (173 KL).

Hôte: Tupaia glis (Diard).

Localisation : Intestin.

Origine GÉOgRAPHIQUe: Sepilok, 15 miles de Sandakan, 18-1-74. Bornéo.

L'espèce se trouve chez tous les Tupaia autopsiés dans la même région : 4 Tupaia glis (164 KL, $173 \mathrm{KL}, 191 \mathrm{KL}, 205 \mathrm{KL})$ et 2 Tupaia tana Raffles (177 KL, $190 \mathrm{KL}$ ). Elle a été trouvée 3 fois dans les poumons en même temps que dans l'intestin (173 KL, $191 \mathrm{KL}, 205 \mathrm{KL})$. Dans l'intestin, comme dans les poumons, elle coexiste généralement avec Anoplostrongylus (Tupaiostrongylus) liei (Dunn, 1963).

\section{Deseription.}

Petits Nématodes, dont le corps est relativement large dans sa région antérieure $(300 \mu$ chez le $\delta, 425 \mu$ chez la $\$$ ), alors que la région postérieure n'atteint que $250 \mu$ chez le $\delta, 350 \mu$ chez la $\uparrow$, juste en avant de la vulve.

La tête présente une petite vésicule céphalique plus large que haute. En vue apicale, elle porte 2 amphides, 4 papilles labiales externes et 4 papilles céphaliques. Il n'existe pas de capsule buccale; la partie antérieure des 3 lobes œsophagiens fait saillie au-dessus du niveau de la bouche ; celle-ci est de forme grossièrement triangulaire (fig. 1, B).

Synlophe: le synlophe, identique pour les 2 sexes, est constitué de crêtes cuticulaires, obliques d'avant vers l'arrière et des axes médians vers les axes latéraux. Les crêtes se présentent sous forme de lignes interrompues, déterminant des rectangles plus ou moins allongés. (fig. 1, A).

Les crêtes naissent de chaque côté des lignes ventrale et dorsale ; très obliques à leur naissance, elles deviennent longitudinales à proximité de la ligne latérale. Elles «s'entassent donc progressivement sur chaque champ latéral, les crêtes les plus proches de l'axe latéral correspondant à celles qui ont une naissance plus antérieure. 
Les naissances de crêtes s'effectuent à intervalles à peu près réguliers sur toute la longueur du corps, depuis le niveau du pore excréteur jusqu'à environ $500 \mu$ de la pointe caudale.

Nous avons compté $4 \times 185$ naissances de crêtes sur une grande femelle. Chaque crête disparaît après un trajet longitudinal d'environ $1,3 \mathrm{~mm}$. Une coupe transversale dans la partie moyenne intéresse 74 arêtes (fig. 1, D).

MÂle : chez un ơ long de $11,2 \mathrm{~mm}$, la vésicule céphalique est haute de $50 \mu$ sur $65 \mu$ de large. Anneau nerveux, pore excréteur et deirides situés respectivement à $195 \mu, 185 \mu$ et $285 \mu$ de l'apex. Esophage long de $450 \mu$.

Bourse caudale symétrique présentant un petit lobe dorsal, figurée en $1 \mathrm{M}$. Papilles pré-bursales situées à $100 \mu$ en avant de la bourse caudale (fig. 1, F).

Spicules ailés, sub-égaux, longs de $420 \mu$. Leur extrémité est bifide (fig. 1, G, H). Chaque branche externe se prolonge par une petite formation triangulaire, en vue ventrale (fig. $1, H$ ), la branche interne étant un peu plus courte et contournée sur elle-même. Gubernaculum haut de $120 \mu$ sur $58 \mu$ dans sa partie la plus large (fig. 1, $N)$.

Cône génital bien développé portant une papille zéro, triangulaire, à extrémité aiguë et 2 papilles sept, allongées, enfermées dans une sorte de sac membranaire (fig. $I, L, 0$ ).

Femelle : chez une $q$ longue de $15 \mathrm{~mm}$, la vésicule céphalique est haute de $50 \mu$ sur $70 \mu$ de large. Anneau nerveux, pore excréteur et deirides situés respectivement à $180 \mu, 170 \mu$ et $240 \mu$ de l'apex. Esophage long de $490 \mu$ (fig. 1, A).

Didelphie. La vulve s'ouvre à $2,75 \mathrm{~mm}$ de la pointe caudale. Vagina vera long de $100 \mu$. Les 2 branches de l'ovéjecteur sont de même longueur: vestibule: $110 \mu$; sphincter : $40 \mu$; trompe : $180 \mu$. La branche utérine antérieure mesure $2,7 \mathrm{~mm}$, la branche postérieure $1,95 \mathrm{~mm}$. Les 2 branches contiennent de nombreux œufs au stade morula, hauts de $62 \mu$ sur $32 \mu$ de large (fig. 1, E).

Queue longue de $70 \mu$, portant en plus de l'appendice caudal 6 pointes caudales. Ce chiffre est le plus général et correspond à une pointe dorsale dédoublée et 2 pointes latéro-ventrales dédoublées. Parfois, une seule ventrale se dédouble (5 pointes), ou chaque ventrale reste simple (4 pointes) (fig. $1, I, J, K$ ).

\section{Discussion.}

Ce Nématode est une forme relique bien typique, car il associe des caractères très primitifs (pointes caudales de la femelle, bourse caudale du mâle) à des caractères très spécialisés (synlophe). La queue de la femelle, armée de fortes pointes caudales, et la tête, dépourvue d'armature chitinoïde, permettent, d'après la nomenclature de Chabaud, 1960, de ranger l'espèce dans les Strongylacanthidae. Anoplostrongylinae; les genres qui paraissent morphologiquement les plus proches sont Anoplostrongylus Boulenger, 1926, parasite de Chiroptères en Amérique du Sud, et 


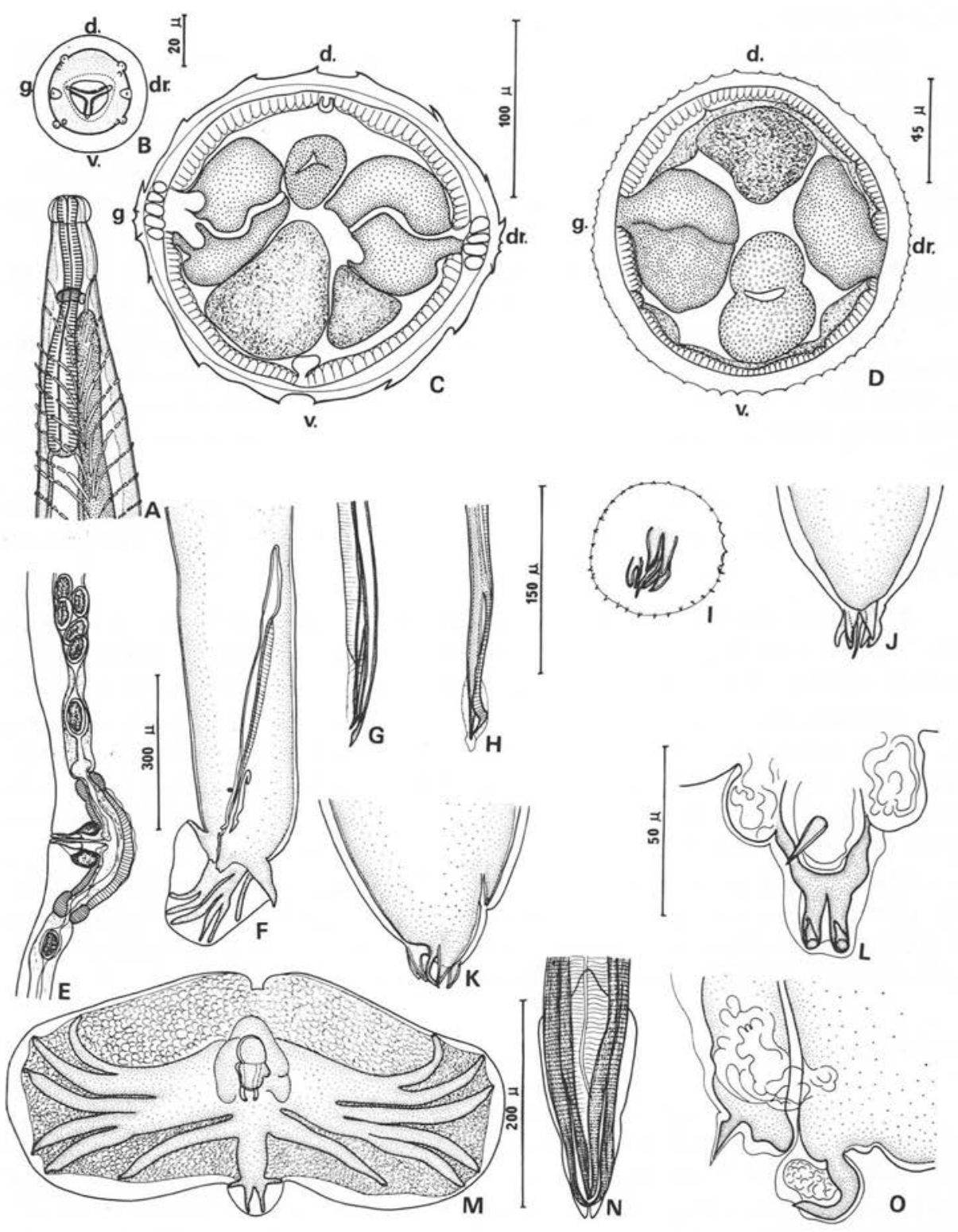


surtout Nycteridostrongylus Baylis, 1930, parasite de Chiroptères en Australie, retrouvé par Meszaros, 1973, au Viêt-Nam.

Les Anoplostrongylinae apparaissent comme un groupe très primitif qui s'est conservé chez les Chiroptères. Il existe des formes de transition, parasites de Mammifères archaïques entre les Anoplostrongylinae et les Molineinae.

Cependant, à l'exception de Bradypostrongylus Price, 1928, qui est insuffisamment connu, les genres Fontesia Travassos, 1928, Pithecostrongylus Lubimov, 1930, Trichochenia Kou, 1958, ont été reclassés dans les Molineinae (cf. Chabaud et coll., 1966) et le genre Nicollina Baylis, 1930, parmi les Amidostomatidae (cf. Inglis, 1968). Donc, à l'exception du genre Tupaiostrongylus Dunn, 1963, qui est précisément parasite de Tupaia, tous les Strongylacanthidae sont parasites de Chiroptères.

Il est particulièrement intéressant de constater que le synlophe du genre Nycteridostrongylus est comparable à celui de notre espèce, car Baylis décrit des crêtes cuticulaires étendues obliquement du dos et du ventre vers les lignes latérales. Mais il nous semble nécessaire de créer un sous-genre particulier, Petiellus n. sub. gen., car l'espèce du Tupaia est dépourvue de formations chitinoïdes buccales et n'a pas l'appareil de fixation formé à partir de la cuticule cervicale ventrale qui existe chez l'espèce du Chiroptère.

Nous la nommons Nycteridostrongylus (Petiellus) petersi n. sub. gen., n. sp., en l'honneur du $\mathrm{P}^{r}$ W. Peters, et nous en donnons la définition suivante :

Nycteridostrongylus avec tête dépourvue de formations chitinoïdes. Absence de crête cuticulaire cervicale ventrale. Parasite de Tupaiidae. Espèce type unique : Nycteridostrongylus (Petiellus) petersi.

\section{Anoplostrongylus (Tupaiostrongylus) liei (Dunn, 1963) n.cb.}

L'espèce a été retrouvée :

1) A Ulu Gombak FR Selangor, Malaisie; dans l'intestin de 7 Tupaia glis $(122 \mathrm{KL}$ à $125 \mathrm{KL}, 161 \mathrm{KL}, 260 \mathrm{KL}, 263 \mathrm{KL})$; dans l'intestin d'un Tupaia minor Günther (126 KL).

FIG. 1. - Nycteridostrongylus (Petiellus) petersi n. sub. gen, n. sp. A : rieure, vue latérale droite. $\mathrm{B}: \hat{\delta}$, tête en vue apicale. $\mathrm{C}: \hat{q}$, coupe transversale au niveau de l'œsophage et des glandes excrétrices. D: id., au milieu du corps. E: $\$$, région des deux branches de l'ovéjecteur, vue latérale gauche. F: ô, extrémité postérieure, vue latérale gauche. $\mathrm{G}, \mathrm{H}$ : $\hat{\delta}$, pointe du spicule gauche successivement vue latérale et vue ventrale. I: $\$$, pointe caudale, vue postérieure. Pointe dorsale grande et dédoublée, pointes ventro-latérales plus petites, bifides. $\mathbf{J}: i d$., vue dorsale. $\mathrm{K}$ : autre ${ }_{+}$, extrémité postérieure, vue latérale droite. $\mathrm{L}$ : $\hat{\delta}$, cône génital, vue ventrale. $\mathbf{M}$ : $\hat{\jmath}$, bourse caudale, vue ventrale. $\mathrm{N}: \hat{\delta}$, gubernaculum en place, vue ventrale. $\mathrm{O}$ : $\hat{\delta}$, cône génital, vue latérale gauche.

A, E, F : $300 \mu ;$ B : $20 \mu ; \mathrm{C}, \mathrm{N}: 100 \mu ; \mathrm{D}: 75 \mu ; \mathrm{G}, \mathrm{H}, \mathrm{I}, \mathrm{J}, \mathrm{K}$ :

$$
150 \mu ; \mathrm{L}, \mathrm{O}: 50 \mu ; \mathrm{M}: 200 \mu \text {. }
$$


2) A 15 miles de Sandakan-Sepilok, Bornéo; dans l'intestin et les poumons d'un Tupaia glis (191 KL), coparasite de Nycteridostrongylus (Petiellus) petersi; dans l'intestin de 2 Tupaia tana (190 KL et $177 \mathrm{KL})$; chez ce dernier, l'espèce est coparasite de $N$. (P.) petersi.

\section{Morphologie :}

Petits Nématodes dont la tête présente une petite vésicule céphalique plus large $(25 \mu)$ que haute $(20 \mu)$. En vue apicale, la tête porte 2 amphides, 4 papilles labiales externes et 4 papilles céphaliques. La partie antérieure des 3 lobes œsophagiens fait saillie au-dessus du niveau de la bouche qui est de forme grossièrement triangulaire (fig. 2, E). Il n'y a pas de capsule buccale.

Pore excréteur situé en avant de l'anneau nerveux. Il est suivi par un très long canal excréteur. Les glandes excrétrices, bien visibles, sont de taille inégale, la droite étant plus importante que la gauche (fig. 2, $C, F$ ). Les deirides, difficiles à observer, sont situées assez postérieurement, entre le début des glandes excrétrices et la fin de l'œsophage (fig. 2, $A, B$ ).

Synlophe: chez les deux sexes, le corps est parcouru longitudinalement par des crêtes cuticulaires dont le nombre augmente d'avant en arrière. Les crêtes naissent le long des lignes médianes et s'étendent jusqu'en avant de la bourse caudale chez le $\delta$ et de l'anus chez la $\$$.

Dans la région antérieure du corps, les crêtes sont au nombre de 20, dont 8 ventrales, 8 dorsales et 2 en face de chaque champ latéral. Les crêtes sont soutenues par des formations chitinoïdes qui déterminent 2 ailes latérales (fig. 2, F). Au milieu du corps, les ailes sont beaucoup moins marquées et il existe 13 crêtes dorsales et 15 crêtes ventrales (fig. 2, H). Dans la partie postérieure du corps, sur une longueur d'environ $180 \mu$ chez le $\delta$, et entre la vulve et l'anus chez la $\leftarrow$, les crêtes sont à peine marquées, mais il existe des ponctuations (fig. 2, D) qui, en coupe transversale, apparaissent comme des prolongements de l'hypoderme (fig. 2, G). En coupe transversale, la pointe des crêtes est orientée perpendiculairement à la paroi du corps.

FIG. 2. - Anoplostrongylus (Tupaiostrongylus) liei (Dunn, 1963). A: ô, extrémité antérieure, vue latérale droite. B: id., vue ventrale montrant la naissance des crêtes cuticulaires. C: $\uparrow$, extrémité antérieure, vue ventrale. D: $\hat{\delta}$, ponctuations sur la partie postérieure du corps, vue ventrale. E: $q$, tête en vue apicale. F: $\uparrow$, coupe transversale du corps passant par les glandes excrétrices au niveau du début de l'intestin. $\mathrm{G}$ : $q$, coupe transversale du corps entre la vulve et l'anus. $\mathrm{H}$ : $ᄋ$, coupe transversale au milieu du corps. I: $\hat{\jmath}$, bourse caudale, vue ventrale. $\mathbf{J}: \hat{\jmath}$, extrémité postérieure, vue ventrale. $\mathrm{K}$ : $\hat{\jmath}$, gubernaculum. $\mathbf{L}: \hat{\jmath}$, pointe d'un spicule, vue latérale. $\mathbf{M}: \uparrow$, pointe caudale étalée, la pointe dorsale est simple, les deux pointes latéro-ventrales sont dédoublées. $\mathrm{N}$ : + , extrémité caudale, vue latérale droite. $\mathrm{O}:$ 으, région des deux branches de l'ovéjecteur, vue latérale gauche.

A, B, C, O: $150 \mu ; \mathrm{D}, \mathrm{E}, \mathrm{F}, \mathrm{G}, \mathrm{H}, \mathrm{M}, \mathrm{N}: 50 \mu ; \mathrm{I}, \mathrm{K}, \mathrm{L}: 30 \mu ; \mathrm{J}: 100 \mu$. 


$$
\begin{aligned}
& \| \mathrm{NO}_{0} \\
& \forall-y
\end{aligned}
$$


MÂle : chez un ồ long de $2,8 \mathrm{~mm}$ et large de $60 \mu$ dans sa partie moyenne, la vésicule céphalique est haute de $30 \mu$ sur $23 \mu$ de large. Anneau nerveux et pore excréteur situés respectivement à $130 \mu$ et $105 \mu$ de l'apex. Esophage long de $235 \mu$ (fig. 2, A).

Bourse caudale symétrique, avec un petit lobe dorsal, figurée en $2, I, J$. Papilles prébursales situées à $40 \mu$ en avant de la bourse caudale (fig. 2,J). Spicules sub-égaux, ailés, longs de $190 \mu$. Extrémité distale bifide avec pointe interne un peu plus courte et recourbée. Gubernaculum long de $48 \mu$ (fig. 2, K). Cône génital peu développé. Papille zéro non vue, papilles sept très petites (fig. 2, I).

Femelle: chez une $q$ longue de $2,9 \mathrm{~mm}$ et large de $75 \mu$ dans sa partie moyenne, la vésicule céphalique est haute de $26 \mu$ sur $28 \mu$ de large. Anneau nerveux et pore excréteur situés respectivement à $180 \mu$ et $155 \mu$ de l'apex. CEsophage long de $250 \mu$ (fig. 2, C).

Didelphie : la vulve s'ouvre à $500 \mu$ de la pointe caudale. Vagina vera long de $30 \mu$. La branche antérieure de l'ovéjecteur comprend un vestibule de $39 \mu$, un sphincter de $26 \mu$ sur $36 \mu$, une trompe de $43 \mu$. La branche utérine antérieure mesure $275 \mu$. La branche postérieure de l'ovéjecteur comprend un vestibule de $21 \mu$, un sphincter de $24 \mu$ sur $37 \mu$, une trompe de $42 \mu$. La branche utérine postérieure mesure $300 \mu$. Les œufs sont hauts de $48 \mu$ sur $22 \mu$ de large. Leur nombre varie de 2 à 6 pour chaque branche utérine. $M N)$.

Queue longue de $40 \mu$, présentant, en plus du filament caudal, 5 pointes (fig. 2

\section{Discussion.}

Cette espèce est extrêmement proche ou identique à Tupaiostrongylus liei (Dunn, 1963), parasite de Tupaia glis sordida dans une île située à 30 miles de la péninsule malaise.

Les différences entre les descriptions (place de l'anneau nerveux, pointe des spicules, pointe caudale de la \&) nous paraissent relever essentiellement de différences d'interprétation et nous pensons pouvoir assimiler nos spécimens à cette espèce.

Nous sommes en plein accord avec l'interprétation générale de Dunn, qui rapproche cette espèce des parasites de Chiroptères et, en particulier, de Molinostrongylus Skarbilovitsch, 1934; malheureusement, l'auteur n'a pas pris en considération le genre Anoplostrongylus Boulenger, 1926. Une révision des Strongylacanthidae, Anoplostrongylinae, parasites de Chiroptères, mériterait d'être faite en s'appuyant sur les caractères fournis par le synlophe. Les nombreux genres, répartis par Skrjabin et coll., 1954, dans des sous-familles variées, paraissent appartenir en réalité à deux groupes dont les représentants les plus synthétiques sont Anoplostrongylus et Molinostron. gylus. D'assez nombreux genres ont été différenciés parce qu'ils ont tel ou tel caractère particulier (ombrelle céphalique, ailes cervicales, taille des tubercules caudaux de la femelle, etc...), alors que les deux genres de base paraissent, à première vue, proches 
l'un de l'autre. Ces derniers ont été différenciés en 1934 par Skarbilovitsch, qui s'est appuyé principalement sur les caractères des spicules, de la côte dorsale et de l'extrémité caudale de la femelle.

Maintenant que l'on connaît de nombreuses espèces, les deux genres apparaissent valides, mais certains Molinostrongylus ayant une côte dorsale très courte (ce qui ne répond pas à la définition de Skarbilovitsch), nous pensons que des caractères différentiels, non encore pris en considération, ont une plus grande valeur.

On constate, en effet, que tous les Anoplostrongylinae parasites de Chiroptères du Nouveau Monde, quels que soient les genres où ils sont classés (Anoplostrongylus Boulenger, 1926, Allintoshius Chitwood, 1937, Cheiropteronema Sandground, 1929, Parallintoshius Araujo, 1940, Torrestrongylus Vigueras, 1935, Tricholeiperia Travassos, 1935), ont une côte 4 plus courte que la côte 3 ; la papille terminale de la côte 4 est située à mi-distance des terminaisons des côtes 3 et 5 ; la cuticule ventrale de la bourse caudale est inerme. Anoplostrongylus delicatus Schwartz, 1928, décrit du Texas, doit donc, à notre avis, rester dans le genre Anoplostrongylus.

Au contraire, les Anoplostrongylinae parasites de Chiroptères de l'Ancien Monde, quels que soient les genres où ils sont classés (Molinostrongylus, Nycteridostrongylus Baylis, 1930), ont tous une côte 4 presque aussi longue que la côte 3 ; la papille terminale de la côte 4 est située près de celle de la 3 ; la cuticule ventrale de la bourse caudale est armée d'épines ou, au moins, ornée de fortes ponctuations.

Chabaud et coll., 1970, ont démontré que les caractères fournis par la côte 4 ont une bonne valeur phylétique et, d'après ce critère, les formes américaines apparaissent plus primitives que celles de l'Ancien Monde.

Paradoxalement, l'espèce du Tupaia doit être rapprochée des parasites de Chiroptères du Nouveau Monde et, de fait, nous voyons si peu de différences entre les genres Anoplostrongylus et Tupaiostrongylus que nous préférons considérer le second comme un sous-genre du premier. En effet, en dehors de l'hôte et de la localisation géographique, nous ne voyons guère que le nombre d'épines caudales de la femelle qui permette de séparer les deux sous-genres. Nous désignons donc nos spécimens sous le nom d'Anoplostrongylus (Tupaiostrongylus) liei (Dunn, 1963) n. comb.

\section{Hepatojarakus malayae Yeh, 1955}

MAtÉriel : -2 ô, 1 , un fragment antérieur $\$$, chez Tupaia tane Raffles, $\mathrm{n}^{\circ}$ 91267. Ranan. Kg Nalapak, Sabah, Bornéo. - 1 $\hat{\delta}$, parasite de l'intestin grêle d'Aeromys thomasi (Petauristinae), $\mathrm{n}^{\circ}$ 2899, originaire de Kinabalu, Bornéo.

\section{Description.}

Petits Nématodes courbés le long de leur ligne ventrale, de diamètre relativement important par rapport à leur longueur. Le corps est fortement dilaté, d'une part, entre les couches musculaires et la cuticule, d'autre part, au niveau de la cavité 
générale, le tube digestif et l'appareil génital n'occupant qu'une faible partie de cette dernière (fig. $3, N$ ). Présence d'une vésicule céphalique presqu'aussi baute que large, mais légèrement plus courte du côté ventral. La tête porte 2 amphides groupées avec les papilles labiales latérales et 4 papilles céphaliques groupées avec les labiales externes sub-médianes. En vue apicale, la bouche, grande, est de forme hexagonale arrondie. Elle est doublée par une couronne de petites denticulations, qui évoque, sous forme atrophiée, la corona radiata des Strongylidae (fig. 3, B).

Synlophe : dans les 2 sexes, le corps est parcouru, du bord de la vésicule céphalique jusqu'à l'extrémité caudale, par des lignes longitudinales, grossièrement parallèles, interrompues de façon irrégulière (fig. 3, A). En coupe transversale, ces lignes apparaissent sous forme de très nombreuses crénelures (185 chez le spécimen ô coupé) (fig. 3, D).

MÂle: corps longs de $6,8 \mathrm{~mm}$, large de $300 \mu$ dans sa partie moyenne. Vésicule céphalique haute de $60 \mu$ (côté dorsal) sur $50 \mu$ de large. Anneau nerveux, pore excréteur et deirides situés respectivement à $190 \mu, 235 \mu$ et $240 \mu$ de l'apex. Esophage long de $375 \mu$.

Bourse caudale figurée en $3, L, M$ : côtes 4 courtes; côtes 2 et 3 d'une part, 5 et 6 , d'autre part, rapprochées; cuticule ventrale portant de nombreuses ponctuations. Spicules complexes, ailés, longs de $200 \mu$, divisés en 3 branches. L'extrémité distale de la branche externe est en forme de crochet et recourbée ventralement (fig. 3, J, K). Gubernaculum légèrement concave du côté ventral, haut de $110 \mu$, figuré en $3, I$.

Femelle: corps long de 10,8 mm, large de $320 \mu$ dans sa partie moyenne. Vésicule céphalique haute de $50 \mu$ (côté dorsal) sur $50 \mu$ de large. Anneau nerveux, pore excréteur et deirides situés respectivement à $160 \mu, 215 \mu$ et $210 \mu$ de l'apex. Esophage long de $430 \mu$ (fig. 3, C).

Didelphie: la vulve s'ouvre à $2,6 \mathrm{~mm}$ de la pointe caudale. Vagina vera fortement chitinisé, long de $40 \mu$. Les deux branches de l'ovéjecteur sont de longueur sub-égale : vestibule : $120 \mu$; sphincter : $42 \mu \times 37 \mu$; trompe: $175 \mu$ (fig. $3, E$ ). La branche utérine antérieure mesure $1,9 \mathrm{~mm}$, postérieure, 1,7 mm. Les œufs sont très nombreux et mesurent $50 \mu \times 28 \mu$. Queue longue de $170 \mu$ portant une pointe terminale de $20 \mu$ (fig. 3, F, G).

FIG. 3. - Hepatojarakus malayae Yeh, 1955. A: $q$, détail des crêtes cuticulaires au niveau du pore excréteur et de la deiride droite. B: $\hat{\delta}$, tête en vue apicale. B': $i d .$, vue interne de la coupe frontale. C: + , extrémité antérieure, vue latérale gauche. D: $\hat{\delta}$, coupe transversale au milieu du corps. E: $\uparrow$, région des 2 branches de l'ovéjecteur, vue latérale droite. F: $q$, extrémité caudale, vue ventrale. G: $ᄋ$, queue, vue latérale droite. $\mathrm{H}$ : $\hat{\delta}$, cône génital, vue latérale droite. $\mathbf{I}: \hat{\delta}$, gubernaculum, vue latérale droite. $\mathbf{J}: \hat{q}$, spicule droit, vue latérale droite. $\mathrm{K}: \hat{\delta}$, spicule gauche, vue dorsale. L: $\hat{\delta}$, bourse caudale, vue ventrale (ponctuations non indiquées). $\mathbf{M}: \hat{\delta}$, bourse caudale, lobe gauche avec les ponctuations.

A, B, F, H, K : éch. : $50 \mu$; C, E, G, L : éch. : $150 \mu$; D : éch. : $75 \mu$; I, J, M : éch. : $100 \mu$ 


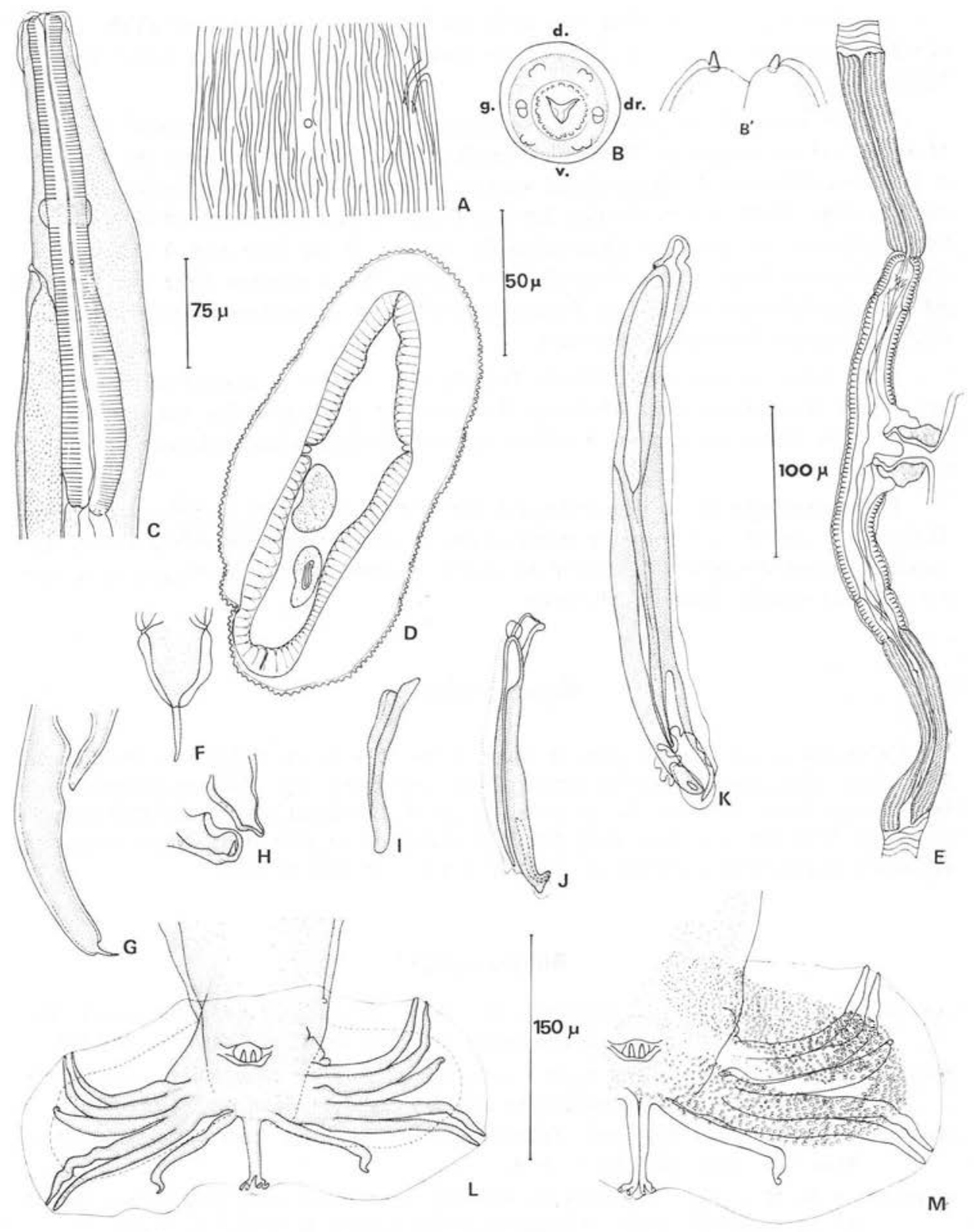




\section{Diseussion.}

L'espèce peut être assimilée sans difficulté à Hepatojarakus malayae (Yeh, 1955). décrit des canaux biliaires et de la veine cave inférieure de Rattus rattus jarak à Malacca.

Il était anormal de trouver un Nématode proche du genre Molineus chez un Muridae, car ce groupe de Nématodes paraît avoir évolué dès le début du Tertiaire et être caractéristique de Mammifères archaïques et non de Rongeurs modernes (Chabaud et coll., 1967). La localisation hors de l'intestin était également un élément très important pour suspecter un phénomène de capture. Il est donc tout à fait satisfaisant de trouver l'espèce dans l'intestin d'un Tupaia. Nous pensons donc que l'espèce est fondamentalement parasite de Tupaia et qu'elle s'est adaptée secondairement à différents Rongeurs Sciuridés et Muridés.

D'un point de vue nomenclature, l'un de nous (1959) a suggéré la synonymie des genres Hepatojarakus et Molineus. Le synlophe très particulier du parasite du Tupaia nous incite aujourd'hui à penser que l'individualisation d'Hepatojarakus est utile.

La morphologie de ce Nématode, qui conserve les caractères archaïques du genre Molineus et même une coronule péribuccale, et qui présente en même temps des caractères hyperspécialisés (dilatation du corps, synlophe), paraît correspondre à une petite lignée relique, issue de Molineus.

\section{REMERCIEMENTS}

Ce travail a été effectué dans le cadre d'une Mission en Malaisie à l'Institut de Recherches Médicales de Kuala-Lumpur. Nous exprimons nos vifs remerciements au $\mathrm{D}^{r}$ Bhawgan Singh, Directeur de cet Institut et au $\mathrm{D}^{r}$ Lim-Boo-Liat, chef du Département d'Ecologie Médicale, pour nous avoir permis la réalisation de cette étude. Nous remercions également le personnel technique du laboratoire pour son aide efficace.

\section{Bibliographie}

Araujo (T.-L. de), 1940. - Parallintoshius parallintoshius n.g.n. sp. (Nematoda Trichostrongylidae) parasita de Chiroptera. Rev. Facult. Medic. veter., 1, 205-210.

Boulenger (C. L.), 1926. - Report on a collection of parasitic Nematodes, mainly from Egypt. Part IV. Trichostrongylidae and Strongylidae. Parasitology, 18, 86-100.

BAYLIS (H. A.), 1930. - Four new Trichostrongylid Nematodes from Queensland. Ann. Mag. Nat. Hist., sér., 10, 6, 1-18.

Cameron (T. W. M.), 1923. - Studies on two new genera and some little known species of the nematode family, Trichostrongylidae Leiper. J. Helm., 1, 71-96.

Снabaud (A.-G.), 1960. - Remarques sur la systématique des Nématodes Trichostrongyloidea. Bull. Soc. Zool. de France, 84 (1959), 473-483. 
Chabaud (A.-G.), Bain (O.) et Puylaert (F.), 1967. - Description de trois nouveaux Nématodes Molineinae et considérations sur la systématique et le caractère archaïque de cette sous-famille. Bull. Mus. Nat. Hist. Nat., $2^{\circ}$ sér., 38, 904-920.

Chabaud (A.-G.), Puylaert (F.), Bain (O.), Petter (A.-J.) et Durette-Desset (M.-C.), 1970. - Remarques sur l'homologie entre les papilles cloacales des Rhabditides et les côtes dorsales des Strongylida. C.R. Acad. Sc. (Paris), 271, sér. D, 17711774 .

Chitwood (B.-G.), 1937. - A new trichostrongyle Allintoshius nycticeius n. g. n. sp. (Nematoda) from a bat. Proc. Helm. Soc. Washington, 4, 19-20.

Dunn (F. L.), 1963. - A new Trichostrongylid Nematode from an Oriental Primate. Proc. Helm. Soc. Washington, 30, 161-165.

INGLIS (W. G.), 1968. - The geographical and evolutionary relationships of Australian trichostrongyloid parasites and their hosts. J. Linn. Soc. (Zool.), 47, 327-347.

Kou (C. C.), 1958. - Studies on parasitic nematodes of mammals from Canton. Acta Zoologica Sinica, 10, 79-82.

Lubimov (M. P.), 1930. - Pithecostrongylus satyri n. g. n. sp. trouvé dans l'intestin d'un Orang-Outang. Ann. Parasit. hum. comp., 8, 51-56.

Meszaros (F.), 1973. - Parasitic Nematodes of bats in Vietnam I. Parasit. Hung., 6, 149-167.

Price (E. W.), 1928. - Two new nematode worms from rodents. Proc. U.S. Nat. Mus., $74,1-5$.

Schwartz (B.), 1927. - A new parasitic nematode from an unknown species of bat. Proc. U.S.N.M., 71, 1-4.

SANDGround (J. H.), 1929. - Some new parasitic Nematodes from Yucatan (Mexico) including a new genus of Strongyle from cattle. Bull. Mus. Comp. Zool. Harvard, 69, 515-524.

SKarbilovitsch (T.-S.), 1934. - Sur la faune des Trichostrongylidae des chauve-souris. Ann. Parasit. hum. comp., 12, 350-361.

Skrjabin (K.-I.), Schikhobalowa (N.-P.) et Schulz (R.-S.), 1954. - Osnovi Nematodologi, IV, Dictyocaulides et Héligmosomatides, 1-323.

Travassos (L.), 1928. - Trichostrongylidae do Tamandua tetradactyla (L.). Bol. Biol., $11,23-40$.

Travassos (L.), 1935. - Alguns novos generos e especies de Trichostrongylidae. Rev. Med. Cirurg. Brasil, 43, 345-361.

Vigueras (I.-P.), 1935. - Torrestrongylus torrei n. gen. n. sp. parasito de Chiroptera. Mem. Soc. Cubana Hist. Nat. Habana, 9, 57-58.

YEH (L.-S.), 1955. - A new bursate Nematode Hepatojarakus malayae gen. et sp. nov. from the liver of Rattus rattus jarak (Bonhote) on Pulau Jarak, Straits of Malacca. J. Helminth., 24, 44-48. 